\title{
CORRECTION
}

\section{Correction: Breast cancer management pathways during the COVID-19 pandemic: outcomes from the UK 'Alert Level 4' phase of the B-MaP-C study}

Rajiv V. Dave (D), Baek Kim, Alona Courtney, Rachel O'Connell, Tim Rattay, Vicky P. Taxiarchi, Jamie J. Kirkham, Elizabeth M. Camacho, Patricia Fairbrother, Nisha Sharma, Christopher W. J. Cartlidge, Kieran Horgan, Stuart A. Mclntosh (ID, Daniel R. Leff, Raghavan Vidya, Shelley Potter (D), Chris Holcombe, Ellen Copson (D), Charlotte E. Coles, Ramsey I. Cutress, Ashu Gandhi,

Cliona C. Kirwan and on behalf of the B-MaP-C study collaborative

British Journal of Cancer (2021) 125:772; https://doi.org/10.1038/s41416-021-01378-x

Correction to: British Journal of Cancer https://doi.org/10.1038/s41416020-01234-4, published online 25 March 2021

The original version of this article unfortunately contained a mistake. Due to a typesetting error one of the corresponding authors was omitted. Both, Rajiv V. Dave and Cliona C. Kirwan are corresponding authors of this paper. We apologize for this mistake. The original article has been corrected. 